\title{
Alamethicin Channels as a Signal Transduction Element in an Immobilized Single Giant Unilamellar Vesicle
}

\author{
Hiroyuki Fukuda, ${ }^{1}$ Masaki Nitta, ${ }^{1}$ Misato Sakamoto, ${ }^{1}$ \\ Atsushi Shoji, ${ }^{2 *}$ and Masao Sugawara ${ }^{1}$ \\ ${ }^{1}$ Department of Chemistry, College of Humanities and Sciences, Nihon University, \\ Sakurajousui, Setagaya, Tokyo 156-8550, Japan \\ ${ }^{2}$ Tokyo University of Pharmacy and Life Science, 1432-1 Hachioji, Horinouchi, Tokyo 192-0392, Japan
}

(Received August 31, 2020; accepted December 2, 2020)

Keywords: single giant unilamellar vesicle, alamethicin channel, bovine serum albumin, avidin slip

A single giant unilamellar vesicle (GUV) functionalized with an anti-bovine serum albumin (BSA) antibody was immobilized on an avidin slip, and alamethicin channels were embedded as a signal transduction element for creating a channel-based molecular sensing system. The GUV sensor based on the membrane-bound anti-BSA antibody receptor exhibited alamethicin activities that reflected the binding of BSA (an analyte) at the membrane/solution interface. The normalized integrated current at $-60 \mathrm{mV}$ was able to be used as a measure of the amount of BSA in a solution. The quantification of BSA at $\mathrm{pg} / \mathrm{mL}$ level was demonstrated.

\section{Introduction}

Planar bilayer membranes (planar BLMs), especially free-standing ones, and vesicular bilayer membranes such as uni- or multilamellar liposomes, which are often functionalized with receptors, have a variety of applications in biochemical and biosensing studies. ${ }^{(1-8)}$ However, vesicular bilayer membranes are not able to record channel currents, except for patch-clamped giant unilamellar vesicles (GUVs) of above $10 \mu \mathrm{m}$ diameter. On the other hand, GUVs can be imaged using a variety of optical microscopic approaches and, hence, the manipulation of a single GUV is relatively easy. If channels or related compounds are reconstituted into chemically defined GUVs, they will provide a useful platform for the development of a highly sensitive molecular sensing system, because a spherical membrane has a volume of fL level and allows us to incorporate a large number of channels. However, the application of GUVs to channel-based sensing systems has not been reported, although a limited number of GUVs have been found to have applications in electrophysiological recordings based on the patch-clamp technique. ${ }^{(9-13)}$

For the design of channel-based sensors, we have shown that the distortion of bilayer lipid membranes caused by the antibody-antigen interaction at the bilayer/solution interface results in the modulation of the monomer/dimer kinetics of gramicidin A in the membrane. ${ }^{(14-19)}$ This leads to changes in the integrated channel currents ${ }^{(14-17)}$ and fluorescence intensities ${ }^{(18,19)}$ of

${ }^{*}$ Corresponding author: e-mail: ashoji@toyaku.ac.jp

https://doi.org/10.18494/SAM.2021.3067 
the membrane, whose magnitudes depend on the concentration of an analyte in a solution. Such membrane-bound receptor approaches using peptide channels are promising for the development of highly sensitive electrochemical and optical sensing systems.

In the present study, the potential of alamethicin as a signal transduction element in a channel-based GUV sensor is demonstrated with anti-bovine serum albumin (BSA) antibody (anti-BSA) as a receptor and BSA as a model analyte in the membrane-bound receptor approach. The channel properties of alamethicin ${ }^{(20-29)}$ in bilayer membranes have been extensively studied, but its use as a signal transduction element in a GUV-based sensor has not been reported. Immobilizing GUVs on a chemically modified solid support improves the success probability of clamping a single GUV, and can easily be adapted to a flow system, leading to the easiness of washing unreacted compounds, uninserting channel molecules into bilayer membranes, and exchanging buffer solutions.

\section{Materials and Methods}

\subsection{Reagents}

Alamethicin and albumin from bovine serum (BSA, >97\%) were obtained from SigmaAldrich Chemical Co. (St Louis, MO). Gramicidin A was obtained from Sigma-Aldrich Chemical Co. 1,2-Diphytanoyl-sn-glycero-3-phosphocholine (DPhPC, $10 \mathrm{mg} / \mathrm{mL}$ chloroform solution), 1-2-dioleoyl-sn-glycero-3-phosphoethanolamine (DOPE, $10 \mathrm{mg} / \mathrm{mL}$ chloroform solution), and 1,2-dioleoyl-sn-glycero-3-phosphoethanolamine- $N$-(cap biotinyl) (sodium salt) (B-cap-PE, powder) were purchased from Avanti Polar Lipids, Inc. (Alabaster, AL). Cholesterol (Chol) was obtained from Wako Pure Chemicals Co. (Osaka, Japan) and recrystallized three times from methanol. 2-[4-(2-Hydroxyethyl)-1-piperazinyl]-ethanesulfonic acid (HEPES) and 1-ethyl-3- [3-dimethylaminopropyl]-carbodiimide hydrochloride (EDC) were obtained from Dojindo Laboratories (Kumamoto, Japan). Sorbitol, $\mathrm{N}$-hydroxysuccinimide (NHS), and $\mathrm{N}$-(4maleimidobutyryloxy)sulfosuccinimide sodium salt (sulfo-GMBS) were obtained from Wako Chemicals Co. Sheep polyclonal anti-BSA antibody (anti-BSA) was obtained from Bethl Lab. (TX, USA). 3-Mercaptopropyltrimethoxysilane (MTS, >99.9\%) was obtained from Shin-Etsu Chemical (Tokyo, Japan). 1-Aminoethnolamine was obtained from Tokyo Kasei Co. (Tokyo, Japan). Other reagents used were all of analytical reagent grade. Milli-Q water (Millipore reagent water system, Bedford, $\mathrm{MA}$ ) was used throughout the experiments. $\mathrm{A} \mathrm{Ca}^{2+}, \mathrm{Mg}^{2+}$-free artificial cerebrospinal fluid $\left(\mathrm{Ca}^{2+}, \mathrm{Mg}^{2+}\right.$-free ACSF) containing $0.12 \mathrm{M} \mathrm{NaCl}, 3.0 \mathrm{mM} \mathrm{KCl}$, $23 \mathrm{mM} \mathrm{NaHCO}_{3}, 1.2 \mathrm{mM} \mathrm{NaH}_{2} \mathrm{PO}_{4}$, and $11 \mathrm{mM}$ D-glucose was prepared.

\subsection{Preparation of GUVs and immobilization of a factionalized single GUV on an avidin slip}

GUVs were prepared from a lipid mixture consisting of DPhPC, Chol, DOPE, and B-cap$\mathrm{PE}$ in chloroform at a molar ratio of 9:1:0.050:0.025 by the electroformation method ${ }^{(30-33)} \mathrm{using}$ Vesicle Prep Pro (VPP) (Nanion Technologies, GmbH, Germany). Twenty microliters of the 
lipid mixture in chloroform was placed on an indium tin oxide (ITO)-coated slide glass and air-dried to form a lipid film. After setting a silicon O-ring on the film, $0.30 \mathrm{~mL}$ of $1 \mathrm{~mol} / \mathrm{L}$ sorbitol in Milli-Q water was added. Then an electric field (5 Hz, ac $3 \mathrm{mV})$ was applied through two ITO-coated slide glasses for $2 \mathrm{~h}$. The GUV suspension was recovered and stored in a microcentrifuge tube $(1.5 \mathrm{~mL})$.

The GUV suspension was functionalized with a receptor (anti-BSA) as described below, and then its given portion was used for immobilizing a single GUV on an avidin slip. ${ }^{(18,19)}$ Briefly, $45 \mu \mathrm{L}$ of GUVs (vide supra) was mixed with $30 \mu \mathrm{L}$ of $10 \mu \mathrm{g} / \mathrm{mL}$ anti-BSA in Milli-Q water. Then, a mixture $(30 \mu \mathrm{L})$ of $0.3 \mathrm{~mol} / \mathrm{L}$ NHS and $0.12 \mathrm{~mol} / \mathrm{L}$ EDC in Milli-Q water was added and incubated for $15 \mathrm{~min}$. The activated GUVs were transferred onto an avidin slip set in a flow chamber and incubated for $5 \mathrm{~min}$. Then, a $\mathrm{Ca}^{2+}, \mathrm{Mg}^{2+}$-free ACSF was run at a flow rate of 0.20 $\mathrm{mL} / \mathrm{min}$ for $15 \mathrm{~min}$ to remove unreacted anti-BSA and excess coupling reagents.

\subsection{Current recordings}

The experimental setup used for preparing a GUV sensor and current recordings is shown schematically in Fig. 1(a). A laboratory-made chamber was used, which allowed us to immerse an avidin slip in a chamber solution and perfuse a bath. $\mathrm{A} \mathrm{Ag}-\mathrm{AgCl}$ electrode served as a reference electrode. Patch pipettes were pulled from borosilicate glass capillaries $(1.5 \mathrm{~mm}$ outer diameter and $0.86 \mathrm{~mm}$ inner diameter, Harvard Apparatus Ltd., Kent, UK) using a three-pull

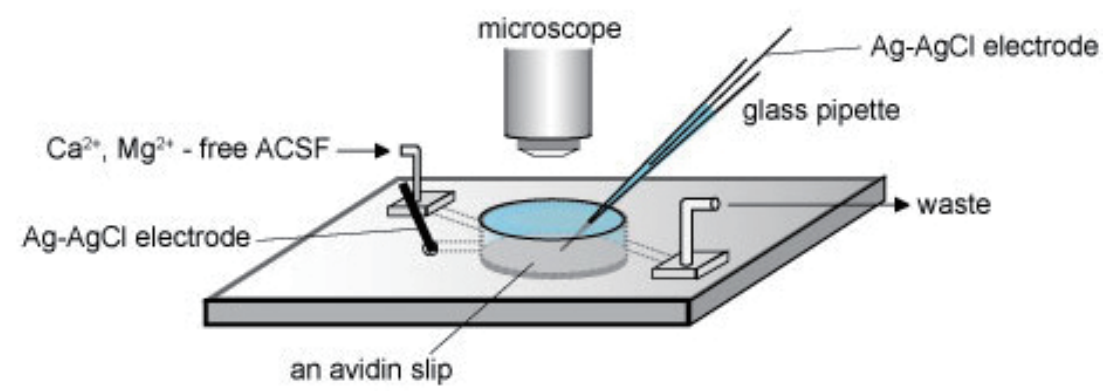

(a)

(1)

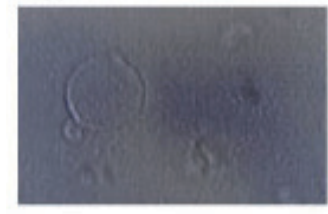

(2)

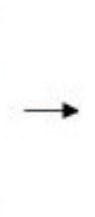

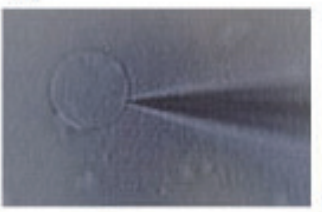

(b)
(3)

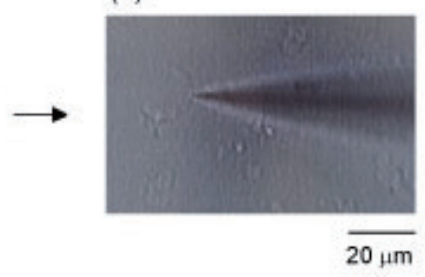

Fig. 1. (Color online) (a) Illustration of a chamber used for preparing a single GUV sensor. The volume of the chamber solution was $0.50 \mathrm{~mL}$. An avidin slip was immersed in the chamber solution. (b) Photos showing the procedure for forming a GUV sensor on an avidin slip. (1) Single GUV in an ACSF. (2) Formation of a gigaseal on a patch pipette. (3) Formation of a single GUV sensor. 
technique with a Sutter micropipette puller (model P-97, Sutter Instrument Co., Novato, CA). The tip size (outer diameter) of the pipette was less than $2 \mu \mathrm{m}$, as observed under a microscope. The pipette (inner) solution was a 10-times diluted $\mathrm{Ca}^{2+}, \mathrm{Mg}^{2+}$-free ACSF containing $5 \mu \mathrm{g} / \mathrm{mL}$ alamethicin. The patch-clamp operations were carried out under a microscope with water immersion objectives (magnification: $40 \times$ ). First, the tip of a patch pipette was kept clean by applying just enough positive pressure above the target GUV [Fig. 1(b), photo 1]. Then, the pipette was pressed on the immobilized GUV to establish a gigaseal [Fig. 1(b), photo 2]. Then, suction was applied to the pipette interior until a sudden increase in the capacitive transient current was observed [Fig. 1(b), photo 3]. Current recordings were performed with an Axopatch 200B patch-clamp amplifier with a built-in $1.0 \mathrm{kHz}$ filter (Axon Instruments Inc., Burlingame, CA). Currents were stored on-line using a PHYSICO PC computer (PHYISICO-Tech Ltd., Tokyo, Japan) in which pCLAMP software (version 8.0; Axon Instruments Inc.) was installed.

Although GUVs of various sizes were present in the ACSF, GUVs of approximately $30-50 \mu \mathrm{m}$ diameter were used for the preparation of GUV sensors because of their easy attachment to a capillary pipette when establishing a gigaseal. Since multichannel responses were expected, recorded channel currents were integrated with respect to time to obtain sensor responses. ${ }^{(8)}$ Furthermore, the magnitude of the integrated current varied from one membrane to another owing to the fact that the number of alamethicin channels embedded in the bilayer membrane was uncontrollable. Thus, the response $(R)$ of the sensor at $-60 \mathrm{mV}$ was the normalized integrated current as follows:

$$
R=\left(I_{B S A}-I_{b}\right) /\left(I_{0}-I_{b}\right)
$$

where $I_{B S A}$ is the integrated current in the presence of BSA, $I_{0}$ is the integrated current in the absence of BSA, and $I_{b}$ is the background current at $0 \mathrm{mV}$.

\section{Results and Discussion}

\subsection{Current recordings}

The first step in the preparation of a patch-clamped single GUV is to attach a glass pipette on the surface of a single GUV to form a tight seal, i.e., the so-called gigaseal, between a patch membrane and a glass pipette. The high seal resistance is essential for recording channel currents of pA level. The success probability of preparing patch sensors was compared between immobilized and suspended GUVs. The achievement of a gigaseal was regarded as the successful attachment of a glass pipette on the bilayer surface of a GUV. For the case of suspended GUVs, the success probability of gigaseal attainment was $35 \%(n=42)$, while that using immobilized GUVs was $55 \%(n=352)$. These results indicate that the immobilization of GUVs is effective for increasing the probability of gigaseal achievement. The lower probability of suspended GUVs was due to the fact that the tip of a patch pipette has to be kept clean by applying a positive pressure before approaching the surface of a targeted GUV. This step often hindered the capture of a single GUV because the GUV was blown off by the pressure. 


\subsection{Voltage-current relationships for alamethicin-incorporated GUVs}

Alamethicin channels are known to be formed in a voltage-dependent and concentrationdependent manner. ${ }^{(20,21,24-26)}$ It has been reported that alamethicin is inserted into membranes when a negative voltage is applied to the trans side of the membrane (peptide addition side), thereby enabling the one-directional insertion of alamethicin. ${ }^{(20)}$ The voltage-current relationship for a single GUV containing alamethicin channels is shown in Fig. 2(a). Multiple

(1)

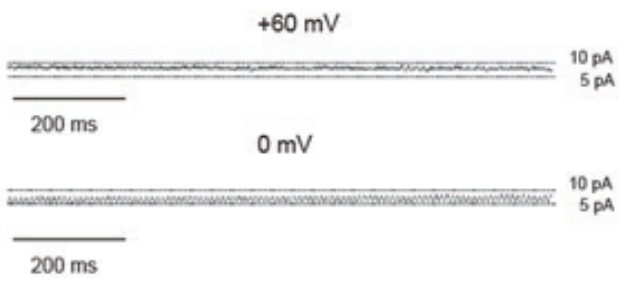

(3)

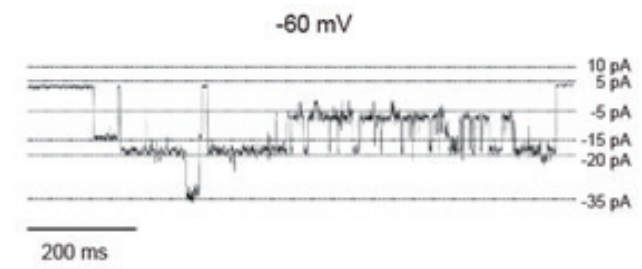

(4)

$-80 \mathrm{mV}$

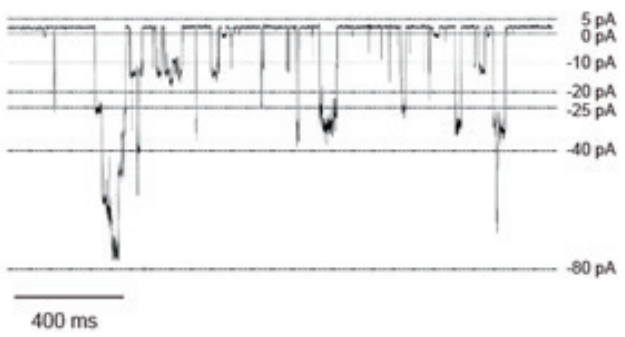

(a)

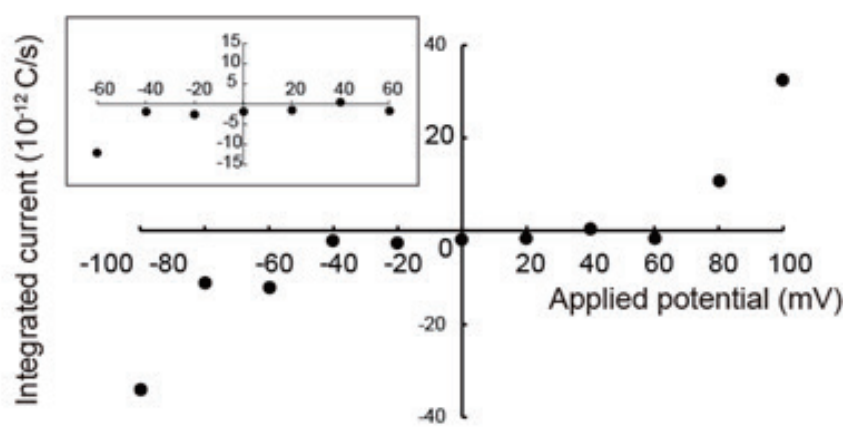

(b)

Fig. 2. (a) Current vs time traces for a single GUV containing alamethicin on an avidin slip in an ACSF solution with applied potentials of (1) +60 , (2) 0 , (3) -60 , and (4) $-80 \mathrm{mV}$. (b) Current vs applied potential relationship. The inset shows an enlargement of the curve in the region between -40 and $+60 \mathrm{mV}$. A single GUV of DPhPC, cholesterol, DOPE, and B-cap-PE (9:1:0.050:0.025 molar ratio) was prepared in $1 \mathrm{~mol} / \mathrm{L}$ sorbitol. The pipette solution was a 10 -times-diluted $\mathrm{Ca}^{2+}, \mathrm{Mg}^{2+}$-free ACSF containing $5 \mu \mathrm{g} / \mathrm{mL}$ alamethicin. 
conductance levels were seen, which are characteristic of alamethicin channels. The plot of the $Q$ vs $V$ relation was asymmetrical, indicating that the extent of translocation of ions was very small at positive potentials of below $+80 \mathrm{mV}$ [Fig. 2(b)], while significant translocation of ions occurred at a negative potential. This suggests that at a negative potential, the onedirectional insertion of alamethicin occurred because alamethicin was added to the pipette solution. At potentials larger than $+80 \mathrm{mV}$, the integrated current increased, seemingly due to the disturbance of the molecular alignment of the bilayer.

Since the composition of electrolytes in a GUV was asymmetric, there is osmotic pressure in a GUV. We observed changes in the diameter of a single GUV (without receptor modification) under a microscope after immobilizing a single GUV on an avidin slip (Fig. 3). In the absence of channels [photo (1)], the GUV exhibited no significant changes in diameter, showing that expansion or shrinkage was negligible. However, in the presence of alamethicin channels [photos (2) and (3)], the GUV diameter increased markedly with increasing concentration of alamethicin. This suggests that the immobilized GUV expanded due to the influx of ions from

(a) aramethicin

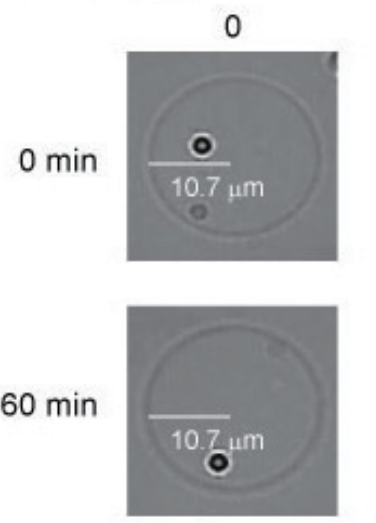

(1)

(b) gramicidin $\mathrm{A}$

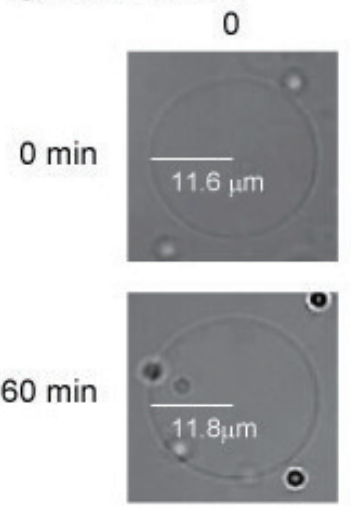

(1)

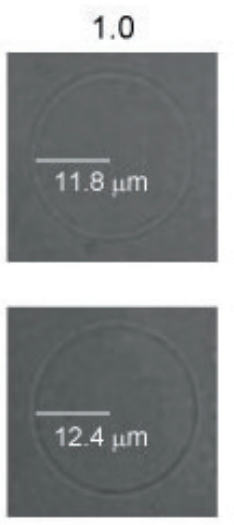

(2)

0.1
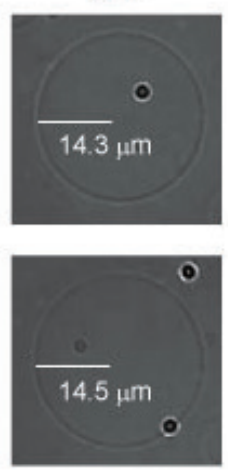

(2)

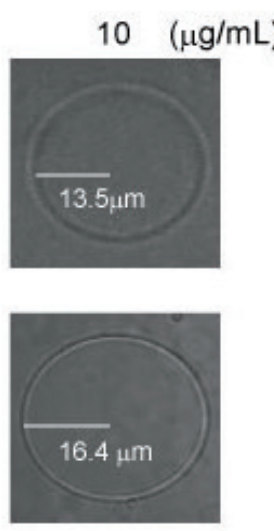

(3)

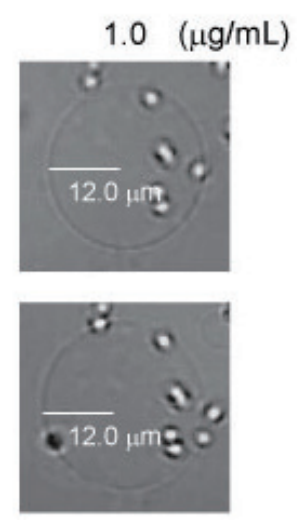

(3)

Fig. 3. Photos of immobilized GUVs in an ACSF solution in the absence and presence of channels. Only relative changes in the diameter with respect to time should be considered because the size of immobilized GUVs differed from one preparation to another. (a) Alamethicin channels. (b) Gramicidin A channels. 
the outer solution to the inner one. No such change in the diameter of a GUV was observed when gramicidin A $(1.0 \mathrm{ng} / \mathrm{mL})$ was used.

\subsection{Detection of BSA with a GUV sensor}

The potential of alamethicin channels as a signal transduction element in a single GUV was investigated by using anti-BSA $(150 \mathrm{kDa}, \mathrm{pI}=8.4)^{(34)}$ as a receptor. The receptor was coupled to the amine moiety of lipid molecules based on the membrane-bound receptor approach. ${ }^{(15-18)}$ Detection of BSA $(69 \mathrm{kDa}, \mathrm{pI}=4.8)^{(35)}$ in an ACSF was investigated by injecting BSA with its final concentration from 1.0 to $5.0 \mathrm{pg} / \mathrm{mL}$.

The current-time profile for the anti-BSA-modified GUV containing alamethicin at $-60 \mathrm{mV}$ (peptide-added side) is shown in Fig. 4. Upon adding BSA, a large negative shift in the current was observed, which was accompanied with a transient shift in the current. The observed currents were multichannel ones; therefore, the integrated channel approach $^{(8)}$ was used to obtain the sensor responses. The integrated current increased with the concentration of BSA in the range from 1.0 to $5.0 \mathrm{pg} / \mathrm{mL}$ [Fig. 4(b), curve 1], while no such changes in the integrated current were observed in the case that receptor (curve 2) and alamethicin channels (curve 3) were absent in the bilayer membranes. These results suggest that alamethicin in the GUV membrane works as a signal transduction element in the membrane-bound receptor approach. The lower detection limit for BSA was approximately $0.06 \mathrm{pg} / \mathrm{mL}$, as calculated from the linear part of the normalized integrated current $(R)$ at three times the standard deviation of $R$ in the absence of BSA.

The selective response of a GUV sensor containing alamethicin was tested with $\gamma$-globulin. The response to $\gamma$-globulin $(50 \mathrm{pg} / \mathrm{mL})(n=3)$ was much smaller than that to BSA $(1.0 \mathrm{pg} / \mathrm{mL})$, indicating that the response was selective to BSA (Fig. 5).

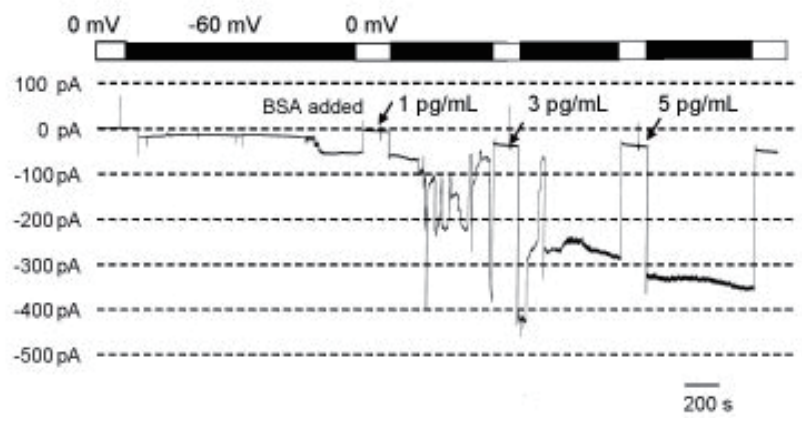

(a)

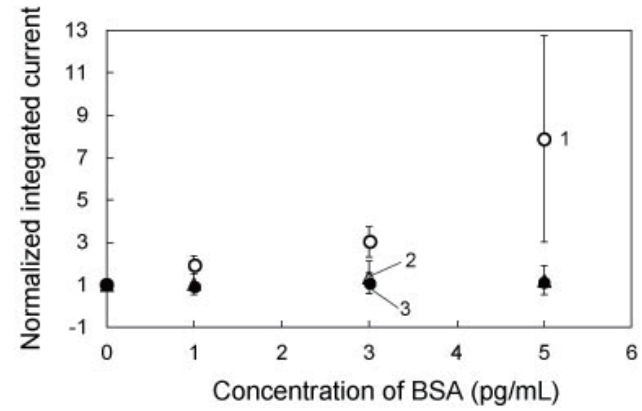

(b)

Fig. 4. (a) Time course of channel currents with a GUV sensor after addition of BSA with a single GUV containing alamethicin channels. The arrows indicate the times at which BSA $(5.0 \mu \mathrm{L})$ was added to a chamber solution $(0.50 \mathrm{~mL})$ at $-60 \mathrm{mV}$ in an ACSF. A single GUV of DPhPC, Chol, DOPE and B-bap-PE (9:1:0.050:0.025, molar ratio) was prepared in $1 \mathrm{~mol} / \mathrm{L}$ sorbitol. The pipette solution was a 10 -times diluted $\mathrm{Ca}^{2+}, \mathrm{Mg}^{2+}$-free ACSF containing $5 \mu \mathrm{g} / \mathrm{mL}$ alamethicin. (b) Concentration dependence for BSA with a single GUV sensor containing alamethicin channels at $-60 \mathrm{mV}$. The response $(R)$ at $-60 \mathrm{mV}$ was normalized using Eq. (1). The average of three measurements was plotted. (1) With anti-BSA antibody, (2) without anti-BSA antibody, (3) without anti-BSA and without alamethicin. 


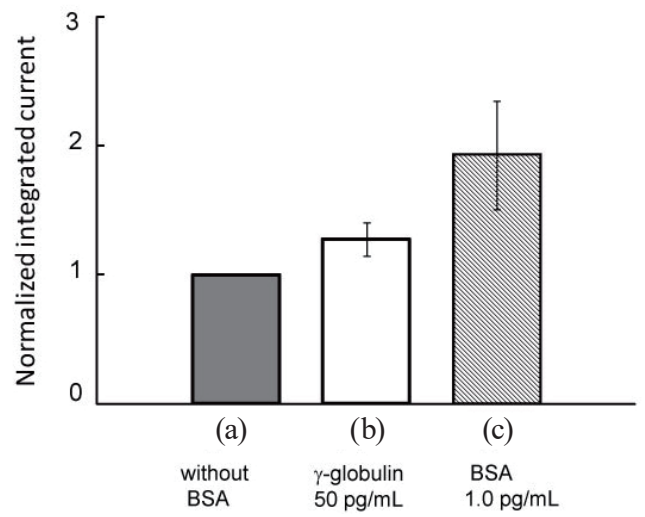

Fig. 5. Effect of $\gamma$-globulin on the response of an alamethicin-based GUV system. The average of three measurements was plotted. (a) Without BSA, (b) with $50 \mathrm{pg} / \mathrm{mL} \gamma$-globulin, (c) with $1.0 \mathrm{pg} / \mathrm{mL}$ BSA.

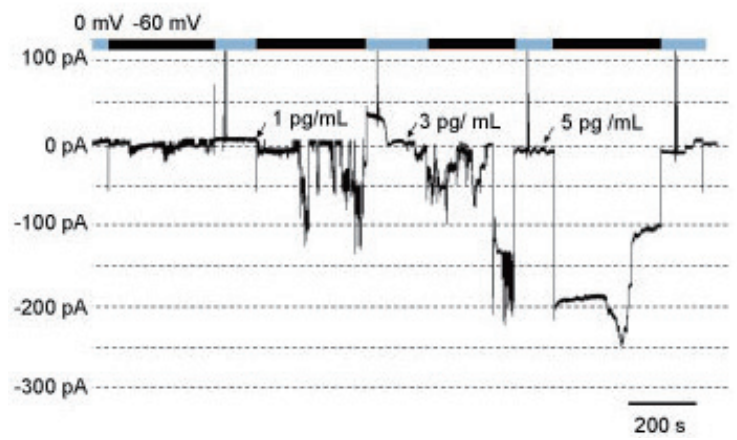

(a)

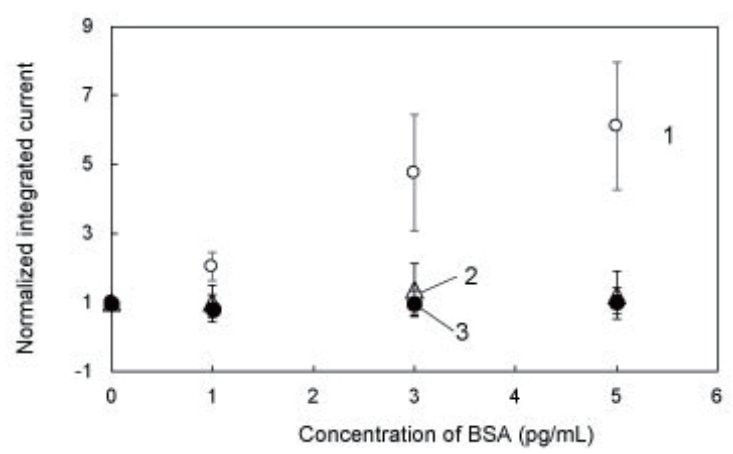

(b)

Fig. 6. (Color online) (a) Time course of channel currents with a GUV sensor after addition of BSA with a single GUV containing gramicidin A channels. The arrows indicate the time at which BSA $(5.0 \mu \mathrm{L})$ was added to a chamber solution $(0.50 \mathrm{~mL})$ at $-60 \mathrm{mV}$ in an ACSF. (b) Concentration dependence for BSA with a single GUV sensor containing gramicidin A channels at $-60 \mathrm{mV}$. The response at $-60 \mathrm{mV}(R)$ was normalized as given in Eq. (1). (1) With anti-BSA antibody, (2) without anti-BSA antibody, (3) without anti-BSA antibody and gramicidin A. The average of three measurements was plotted. A single GUV of DPhPC, Chol, DOPE and B-bap-PE (9:1:0.050:0.025 molar ratio) was prepared in $1 \mathrm{~mol} / \mathrm{L}$ sorbitol. The pipette solution was a 10 -times diluted $\mathrm{Ca}^{2+}, \mathrm{Mg}^{2+}$-free ACSF containing $100 \mathrm{pg} / \mathrm{mL}$ gramicidin A.

\subsection{Alamethicin vs gramicidin}

We considered it worth comparing the response features of a GUV sensor between alamethicin and gramicidin A. The gramicidin A-based GUV exhibited linear concentration dependence for BSA in the range from 1.0 to $5.0 \mathrm{pg} / \mathrm{mL}$ with a detection limit of $0.95 \mathrm{pg} / \mathrm{mL}$ (Fig. 6). On the other hand, the response of the alamethicin-based GUV, especially at concentrations above $5.0 \mathrm{pg} / \mathrm{mL}$ (Fig. 4), exhibited a significant deviation. We suppose that distortion of the alamethicin-embedded bilayer membrane occurred owing to the voltage-dependent insertion of the channel. The better detection limit for the case of alamethicin is attributed to the inherent channel conductance of alamethicin channels, which increases the translocation of ions from the outside solution. 


\section{Conclusions}

The immobilization of a single GUV containing alamethicin channels on an avidin slip is a useful platform for the development of a GUV sensor. Highly sensitive detection of BSA at the $\mathrm{pg} / \mathrm{mL}$ level with a detection limit of sub-pg/mL was achieved by using alamethicin as a signal transduction element. The success probability of the sensor was significantly improved by the immobilization of GUVs on the avidin slip. The present approach will provide a useful way of designing highly sensitive molecular sensing systems.

\section{Acknowledgments}

This work was partially supported by a Grant-in-Aid for Scientific Research (C) (No.21350045) from the Ministry of Education, Culture, Sports, Science, and Technology of Japan. Financial support from a research grant from the College of Humanities and Sciences at Nihon University is also acknowledged.

\section{References}

1 M. Sugawara: Chem. Rec. 18 (2018) 433. https://doi.org/10.1002/tcr.201700046

2 A. Hirano-Iwata, Y. Ishinari, H. Yamamoto, and M. Niwano: Chem. Asian J. 10 (2015) 1266. https://doi. org/10.1002/asia.201403391

3 M. Sugawara, A. Shoji, and M. Sakamoto: Anal. Sci. 30 (2014) 119. https://doi.org/10.2116/analsci.30.119

4 Q. Liu and B. J. Boyd: Analyst 138 (2013) 391. https://doi.org/10.1039/C2AN36140J

5 S. M. Christensen and D. G. Stamou: Sensors 10 (2010) 11352. https://doi.org/10.3390/s101211352

6 B.-W. Park, D.-Y. Yoon, and S.-S. Kim: Biosens. Bioelectron. 26 (2010) 11. https://doi.org/10.1016/ j.bios.2010.04.033

7 A. Hirano, M. Niwano, and M. Sugawara: Trends Anal. Chem. 27 (2008) 512. https://doi.org/10.1016/ j.trac.2008.04.006

8 M. Sugawara, A. Hirano, P. Bühlmann, and Y. Umezawa: Bull. Chem. Soc. Jpn. 75 (2002) 187. https://doi. org/10.1246/bcsj. 75.187

9 S. Aimon, J. Manzi, D. Schmidt, J. A. P. Larrosa, and P. Bassereau: PLOS ONE 6 (2011) 1. https://doi. org/10.1371/journal.pone.0025529

10 L. Kristanc, S. Svetina, and G. Gomišček: Biochim. Biophys. Acta 1818 (2012) 636. https://doi.org/10.1016/ j.bbamem.2011.11.036

11 M. Dezi, A. D. Cicco, P. Bassereau, and D. Levy: Proc. Natl. Acad. Sci. USA 110 (2013) 7276. https://doi. org/10.1073/pnas.1303857110

12 M. S. Collins and S. E. Gordon: J. Vis. Exp. 76 (2013) 1. http://www.jove.com/video/50227

13 I. Motta, A. Gohlke, V. Adrien, F. Li, H. Gardavot, J. E. Rothman, and F. Pincet: Langmuir 31 (2015) 7091. https://doi.org/10.1021/acs.langmuir.5b01173

14 A. Hirano, M. Wakabayashi, Y. Matsuno, and M. Sugawara: Biosens. Bioelectron. 18 (2003) 973. https://doi. org/10.1016/S0956-5663(02)00219-1

15 Y. Matsuno, C. Osono, A. Hirano, and M. Sugawara: Anal. Sci. 20 (2004) 1217. https://doi.org/10.2116/ analsci.20.1217

16 M. Nishio, A. Shoji, and M. Sugawara: Anal. Sci. 28 (2012) 661. https://doi.org/10.2116/analsci.28.661

17 T. Zamoto, S. Tominaga, M. Nishio, A. Shoji, and M. Sugawara: Ana. Sci. 33 (2017) 1421. https://doi. org/10.2116/analsci.33.1421

18 M. Horie H. Yanagisawa, and M. Sugawara: Anal. Biochem. 369 (2007) 192. https://doi.org/10.1016/ j.ab.2007.07.007

19 M. Sakamoto, A. Shoji, and M. Sugawara: Anal. Biochem. 505 (2016) 66. https://doi.org/10.1016/j.ab.2016.04.011

20 A. Futaki, D. Noshiro, T. Kiwada, and K. Asam: Acc. Chem. Res. 46 (2013) 2924. https://doi.org/10.1021/ $\underline{\operatorname{ar} 400051 f}$ 
21 M. Martin, T. Dubbs, and J. R. Fried: Langmuir 33 (2017) 1171. https://doi.org/10.1021/acs.langmuir.6b03309

22 M. Mayer, J. K. Kriebel, M. T. Tosteson, and G. M. Whitesides: Biophys. J. 85 (2003) 2684. https://doi. org/10.1016/S0006-3495(03)74691-8

23 H. S. Le Pioule and K. V. Tabata: Anal. Chem. 80 (2008) 328. https://doi.org/10.1021/ac7016635

24 B. Sakmann and G. Boheim: Nature 282 (1979) 336. https://doi.org/10.1038/282336a0

25 K. H. Steve, J, Ludkle, W. T. Heller, and H. W. Huang: Biophys. J. 71 (1996) 2669. https://doi.org/10.1016/ S0006-3495(96)79458-4

26 O. Muller and D. O. Ruding: Nature 217 (1968) 71. https://doi.org/10.1038/217713a0

27 P. C. Dave, E. Billington, Y. L. Pan, and S. K. Straus: Biophys. J. 89 (2005) 2434. https://doi.org/10.1529/ biophysj. 105.067678

28 D. P. Tieleman, H. J. C. Berendesen, and M. S. P. Sansom: Biophys. J. 80 (2001) 331. https://doi.org/10.1016/ S0006-3495(01)76018-3

29 R. O. Fox. Jr. and F. M. Richards: Nature 300 (1982) 325. https://doi.org/10.1038/300325a0

30 M. L. Angelova and D. S. Dimitrov: Faraday Discuss 81 (1986) 303. https://doi.org/10.1039/DC9868100303

31 M. I. Angelova, S. Soleau, P. Meleard, J. F. Faucon, and P. Bothore: Prog. Coll. Pol. Sci. S. 89 (1992) 127. https://doi.org/10.1007/BFb0116295

32 Y. T. Kang, H. S. Wostein, and S. Majd: Adv. Mater. 25 (2013) 6834. https://doi.org/10.1002/adma.201303290

33 P. Méléard, L. A. Bagatolli, and T. Pott: Method Enzymol. 465 (2009)161. https://doi.org/10.1016/S00766879(09)65009-6

34 B.-S. Lee, S. Gupta, S. Krisnanchettier, and S. S. Lateef: Anal. Biochem. 334 (2004) 106. https://doi. org/10.1016/j.ab.2004.06.041

35 M. E. Soderquist and A. W. Walton: J. Colloid Interface Sci. 75 (1980) 386. https://doi.org/10.1016/00219797(80)90463-4 